Check for updates

Cite this: Phys. Chem. Chem. Phys. 2021, 23, 21521

Received 12th July 2021

Accepted 12th September 2021

DOI: $10.1039 / d 1 c p 03171 f$

rsc.li/pccp

\section{A nanosecond-resolved atomic hydrogen magnetometer $\dagger$}

\author{
Alexandros K. Spiliotis, ${ }^{a b}$ Michalis Xygkis, ${ }^{\text {ab }}$ Konstantinos Tazes, (DD ab \\ George E. Katsoprinakis, ${ }^{\text {ab }}$ Dimitrios Sofikitis, (D) $\ddagger^{\text {ab }}$ Georgios Vasilakis $^{\text {ab }}$ and \\ T. Peter Rakitzis (D) *ab
}

\begin{abstract}
We introduce a novel and sensitive ns-resolved atomic magnetometer, which is at least three orders of magnitude faster than conventional magnetometers. We use the magnetic field dependence of the hyperfine beating of high-density spin-polarized $\mathrm{H}$ atoms, produced from the rapid photodissociation of $\mathrm{HCl}$ gas with sub-ns laser pulses and measured with a pick-up coil, to demonstrate ns-resolved magnetometry, and project sensitivity of a few nT for a spin-projection-limited sensor with $10 \mathrm{nl}$ measurement volume after $1 \mathrm{~ns}$ measurement time. The magnetometer will allow ultrafast continuous $B$-field measurements in many fields, including spin chemistry, spin physics, and plasma physics.
\end{abstract}

\section{Introduction}

Sensitive detection of magnetic fields plays an important role in several fields, including biomedicine, materials science, security screening, geophysical and space surveys, ${ }^{1}$ as well as fundamental physics measurements, such as axion searches. ${ }^{2}$ In addition to the requirement for high sensitivity, numerous applications demand magnetic field detection with high temporal resolution. For instance, ultrafast magnetometry can be used as a sensitive probe of the pathways and kinetics of chemical reactions involving the production of electron or nuclear spin polarization, such as in photo-chemically induced dynamic nuclear polarization (photo-CIDNP). ${ }^{3-6}$ Similarly, studies of magnetodynamics can shed light on dynamical processes in magnetic materials ${ }^{7,8}$ and plasmas, ${ }^{9}$ enabling the development of new technologies. For imaging purposes or for mapping the magnetic field gradients, an important quality is the length scale of the magnetometer, as, in general, smallsized sensors allow for high spatial resolution.

A plethora of magnetometers has been developed to address the challenges in magnetic field sensing (see for example ref. 10 and references therein). Atomic magnetometers based on optically polarized alkali-metal atoms have realized the highest sensitivities, ${ }^{11}$ albeit at low bandwidths, typically up to a few tenths of kHz. ${ }^{12}$ Superconducting quantum interference devices (SQUIDs)

\footnotetext{
${ }^{a}$ Foundation for Research and Technology Hellas, Institute of Electronic Structure and Laser, N. Plastira 100, Heraklion, Crete, GR-71110, Greece. E-mail: ptr@iesl.forth.gr

${ }^{b}$ Department of Physics, University of Crete, Heraklion, Greece

$\dagger$ PACS numbers: 07.55.Ge, 67.65.+z.

\$ Permanent address: Department of Physics, University of Ioannina, Ioannina, Greece.
}

have demonstrated similar sensitivities. Although in principle their frequency response can extend up to a few $\mathrm{GHz}$, practical considerations related to the electronic circuitry for the signal readout reduce their bandwidth to the $\mathrm{MHz}$ range. ${ }^{13}$ Inductive-coil sensors feature high detection-bandwidth and broad applicability to different spin species and environments, but they present relatively low sensitivity compared with other state-of-the-art magnetometers (see however ref. 14 for an ultrasensitive, quantum limited inductive sensor). Optical approaches based on the Kerr effect have realized magnetometry in the picosecond temporal regime; nevertheless, their inherent sensitivity is limited and they are mainly used to probe rather large fields from magnetic materials. ${ }^{7,15}$ Nitrogen-vacancy defects in diamond offer an attractive platform for high spatial resolution..$^{16}$ Yet, the demonstrated sensitivity does not rival alkaliatomic magnetometers or SQUIDs and elaborate dynamic decoupling techniques are required to realize broadband magnetometry.

Here, we present a novel atomic hydrogen magnetometer that can measure magnetic fields with high sensitivity and nanosecond time resolution. The sensitive detection results from realizing large spin densities, many orders of magnitude higher than conventional alkali-metal atomic sensors, while the temporal resolution derives from performing magnetometry at the hyperfine coherences of hydrogen (hyperfine frequency of $1.42 \mathrm{GHz}$ and $0.327 \mathrm{GHz}$ in $\mathrm{H}$ and $\mathrm{D}$, respectively). The proposed magnetometer operates at room temperature, is technically simple to use and can be adjusted to sense sub-micrometer length-scales.

\section{Principle of operation}

High density spin-polarized hydrogen ( $\mathrm{SPH}$ ) is produced by photodissociating hydrohalide gas with a circularly-polarized 
UV laser pulse, by exciting a well-defined dissociative electronic state of the molecule (see ref. 17-19 for details). In hydrohalide photolysis, the fragments acquire a spin-orientation which is correlated to the spin of the dissociating photon, and their final polarization is limited by the dissociation path and by the random direction of the hydrohalide molecular bond with respect to the laser propagation direction. Under readily achieved experimental conditions concerning the laser wavelength and intensity, the photodissociation probability can be near unity, thereby the density of the atomic fragments can be made similar to the initial density of the parent molecule. With this process SPH densities of $10^{19} \mathrm{~cm}^{-3}$ and up to $40 \%$ polarization have been demonstrated for isotropic molecular bonds, ${ }^{18,20,21}$ whereas up to $100 \%$ is possible if the bonds are aligned along the photodissociation-laser polarization axis. ${ }^{22}$

Crucially for the operation of the magnetometer, photolysis is predominantly an electronic process occurring within subpicosecond time-scales, during which the nuclear spin degrees of freedom are effectively frozen. ${ }^{20}$ For hydrogen halides, photodissociation with a sufficiently fast laser pulse typically generates fragments with electronically polarized spins, while the nuclear spins remain in their thermal unpolarized state.

Immediately after the photodissociation (assuming that the $\mathrm{HCl}$ bonds have been aligned parallel to the photodissociation polarization direction, and complete electron polarization), half of the generated $\mathrm{H}$ atoms are in the state

$$
\begin{aligned}
\left|\psi_{0}\right\rangle & =\left|m_{\mathrm{s}}=\sigma / 2, m_{\mathrm{I}}=\sigma / 2\right\rangle_{\mathrm{u}} \\
& =\left|F=1, m_{\mathrm{F}}=\sigma\right\rangle_{\mathrm{c}}
\end{aligned}
$$

and half of the polarized atoms are in the state

$$
\begin{aligned}
\left|\psi_{1}\right\rangle & =\left|m_{\mathrm{s}}=\sigma / 2, m_{\mathrm{I}}=-\sigma / 2\right\rangle_{\mathrm{u}} \\
& =\frac{1}{\sqrt{2}}\left(\left|F=1, m_{\mathrm{F}}=0\right\rangle_{\mathrm{c}}-\sigma\left|F=0, m_{\mathrm{F}}=0\right\rangle_{\mathrm{c}}\right)
\end{aligned}
$$

Here, $F$ is the total spin (sum electronic and nuclear spin) quantum number, $m_{\mathrm{s}}, m_{\mathrm{I}}$ and $m_{\mathrm{F}}$ are respectively the electronic, nuclear and total spin projection along the quantization axis, the subscripts $u$ and $c$ refer to the uncoupled and coupled angular momentum basis respectively, $\sigma$ is the helicity of the photons ( +1 or -1 for $\sigma^{+}$and $\sigma^{-}$respectively), and the quantization axis is taken to be along the laser propagation direction. Unlike $\left|\psi_{0}\right\rangle$, the state $\left|\psi_{1}\right\rangle$ is not an eigenstate of the hyperfine interaction. Atoms prepared initially in $\left|\psi_{1}\right\rangle$ experience hyperfine quantum beats, causing the electron and nucleus to exchange spin at the hyperfine frequency. In the absence of a magnetic field this frequency corresponds to the $21 \mathrm{~cm}$ hydrogen line at $f_{0} \approx 1.42 \mathrm{GHz}$.

A magnetic field parallel to the dissociation axis does not affect to first order the hyperfine coherences resulting from the photodissociation $|0,0\rangle_{\mathrm{c}} \leftrightarrow|1,0\rangle_{\mathrm{c}}$. However, the presence of a magnetic field perpendicular to the pumping direction modifies the hyperfine oscillation and induces a net precession of the spins. Taking the direction of magnetic field as the quantization axis, the evolution of $\left|\psi_{1}\right\rangle$ can be written in the form (ignoring a global phase factor and small amplitude terms of order $\left.\gamma_{\mathrm{H}} B / \omega_{0}\right)$ :

$$
\left|\psi(t)_{1}\right\rangle=\frac{1}{2}\left[\mathrm{e}^{-\imath \omega_{+} t}|1,1\rangle_{\mathrm{c}}^{\prime}+\mathrm{e}^{-\imath \omega_{-} t}|1,-1\rangle_{\mathrm{c}}^{\prime}-\imath \sigma \sqrt{2}|0,0\rangle_{\mathrm{c}}^{\prime}\right],
$$

where $t$ is the time after the photodissociating pulse, the apostrophe distinguishes the quantization axis and the angular frequencies are given by the Breit-Rabi formula:

$$
\omega_{ \pm} \approx \omega_{0} \pm \gamma_{\mathrm{H}} B+\left(\frac{\gamma_{\mathrm{H}} B}{\omega_{0}}\right)^{2},
$$

In the above equation, $\omega_{0}=2 \pi f_{0}, \gamma_{\mathrm{H}} \approx 2 \pi \times 1.4 \mathrm{MHz} \mathrm{G}^{-1}$ is the gyromagnetic ratio of total angular momentum for atomic $\mathrm{H}, B$ is the magnetic field, and we have ignored the small nuclear gyromagnetic Landé factor. Information about the magnetic field can be deduced by monitoring the hyperfine dynamical evolution of the electron spin. In the case of $\mathrm{H}$ this is most easily performed with an inductive pickup coil, which measures the time-dependent ensemble magnetization, determined to a good approximation by the hydrogen electron spin.

Spin decoherence in SPH limits the performance of the magnetometer. The spin decay mechanism originates from depolarizing collisions of $\mathrm{H}$ with the particles in the ensemble, which include the photofragments and their secondary sideproducts from chemical reactions, as well as the parent molecules in the case of partial photodissociation (see ref. 21 and references therein). The impact of collisions to spin-relaxation depends on the density, the conditions of photolysis, and the halide species and a corresponding optimization should be performed according to the requirements for magnetometry. This optimization may involve compromising the SPH density in order to achieve larger coherence times as discussed in ref. 21.

\section{Experiment}

\section{Experimental setup}

We performed an experiment with a DC magnetic field to demonstrate the magnetometer operation. The experimental setup is based on the one described in ref. 20. Briefly, a circularly polarized $150 \mathrm{ps}$ laser pulse at $213 \mathrm{~nm}$ was focused inside a cell filled with 2 bar of high purity $(99.9 \%) \mathrm{HCl}$, as shown in Fig. 1a. A $4 \mathrm{~mm}$ long coil, 4.5 turns, radius of $1 \mathrm{~mm}$ and oriented with its axis along the laser pulse propagation, was employed to measure the time-dependent electron magnetization of the SPH atoms. The pickup coil signal was amplified by an RF amplifier and was recorded by a fast-sampling oscilloscope. The time-constant associated with the induction detector was much smaller than the sampling time, so that the recorded signal was directly proportional to the time derivative of the magnetic flux.

The pumping laser beam was weakly focused to a spot of approximately $100 \mu \mathrm{m}$ in diameter, located at the center of the coil. For the energy of the laser pulse $(3 \mathrm{~mJ})$, the realized intensities were sufficient to dissociate only a small fraction (about $2 \%$ ) of the $\mathrm{HCl}$ molecules inside the beam volume per 

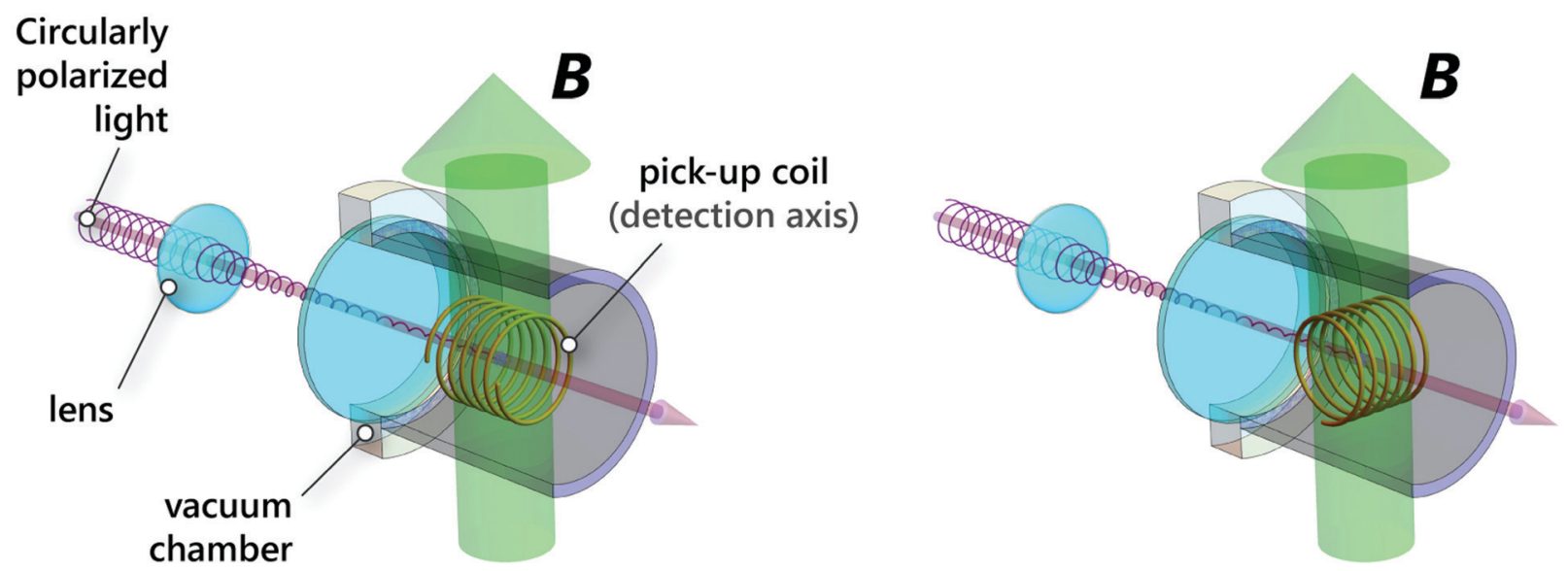

\section{(a) coil axis $\|$ initial polarization $\perp B$}

\section{(b) coil axis $\perp$ initial polarization $\perp B$}

Fig. 1 (a) Experimental setup schematic for the proof of principle demonstration of the magnetometer. A 150 ps, circularly-polarized laser pulse (red arrow), is focused inside a high density $\mathrm{HCl}$ gas cell. A pickup inductive-coil, with its axis parallel to the pumping direction, monitors the ensemble magnetization, while a magnetic field (green arrow) perpendicular to the coil and pumping light is applied; (b) proposed configuration for sensitive detection of time varying magnetic fields. Compared to (a) the detection axis is perpendicular to both the magnetic field and the light pulse.

pulse. Taking into account the $\mathrm{HCl}$ absorption cross section and the photodissociation efficiency we estimate an average SPH density of $10^{18} \mathrm{~cm}^{-3}$ over the coil volume. ${ }^{23}$ Even though this is about $2 \%$ of the parent molecule density, it is more than three orders of magnitude higher than the densities in alkalimetal atomic magnetometers. ${ }^{12}$ For this proof of principle experiment, we traded off SPH density for increased coherence times, as the spin-relaxation cross section for $\mathrm{H}-\mathrm{H}$ and $\mathrm{H}-\mathrm{Cl}$ collisions is much larger than that for $\mathrm{H}-\mathrm{HCl}$ collisions.

We operated the magnetometer in an unshielded environment. This made the pick-up coil and the cables susceptible to electromagnetic interference, originating mainly from discharges in the laser unit. We filtered this noise by subtracting two consecutive measurements acquired with opposite spin orientations in the SPH ensemble. For this, the helicity of the dissociating laser pulse was reversed every laser shot, using a photoelastic modulator (PEM) and an appropriate electronic circuit to phase lock the low frequency laser emission rate with the higher PEM modulation frequency. ${ }^{23}$

A static transverse magnetic field was applied using two permanent round magnets (Neodymium grade N42, $10 \mathrm{~mm}$ diameter and $2 \mathrm{~mm}$ thickness) placed astride the measurement region with their magnetization vectors aligned. The magnets were attached on translation stages, which allowed us to control their separation. This way, a homogeneous, precisely tunable magnetic field, ranging from $10 \mathrm{G}$ to $150 \mathrm{G}$ was applied. A Hall probe was used to calibrate the magnetic field versus magnet separation at low fields, while at higher fields the field was estimated from extrapolation.

\section{Results}

The measured signals, averaged over 50 repetitions, and the corresponding Fourier transforms for various magnetic fields are shown in Fig. 2. The photodissociation pulse occurs at time $t=0$. After a fast transient, which decays at sub-ns timescales, the oscillating signal reflects the $\mathrm{H}$ hyperfine coherences damped by collisions. For zero magnetic field $(B=0)$ a single frequency at $f_{0}$ appears in the Fourier spectrum. A non-zero magnetic field, transverse to the pumping-axis, modifies the frequency of hyperfine coherence and two Fourier peaks appear in the signal, which can be found from eqn (4).

Notice that the heights of the two Fourier peaks $A_{+}$and $A_{-}$ in Fig. 2, which correspond to the frequencies given by 4 , become more unequal with larger $B$ field, and their ratio is given by:

$$
A_{-} / A_{+}=\left(\omega_{-} / \omega_{+}\right) \times \tan ^{2}(\theta)
$$

Eqn (5) has two factors: the ratio of frequencies $\omega_{-} / \omega_{+}$, due to the frequency dependence of coil detection; and the $\tan ^{2}(\theta)$ term, which is the ratio of the square of the projections of the initially prepared state onto the states of the system that beat at $\omega_{-}$and $\omega_{+}$. The angle $\theta$ of the $\tan ^{2}(\theta)$ term is given by $\tan (2 \theta)=$ $1 / \tilde{B}$, where $B=\tilde{B} / B_{\mathrm{c}}$, and $B_{\mathrm{c}}=50.7 \mathrm{mT}$ is the critical field for $\mathrm{H}$ atoms. ${ }^{24}$ Eqn (5) then becomes:

$$
A_{-} / A_{+}=\left(\omega_{-} / \omega_{+}\right) \times\left(2 \tilde{B}^{2}+1-2 \tilde{B} \sqrt{\tilde{B}^{2}+1}\right)
$$

Note that for $\tilde{B}=0, A_{-} / A_{+}=1$, whereas when $\tilde{B}$ tends to infinity, the $A_{-}$peak vanishes, and the ratio $A_{-} / A_{+}$tends to 0 .

In Fig. 3 the measured frequencies are plotted versus the applied magnetic field as quantified from an independent calibration with the Hall probe. The peak frequencies were found using an algorithm which fits a Lorentzian curve to the three consecutive points with the highest amplitudes in the discrete Fourier transform. ${ }^{23}$ The agreement between the frequency peaks and the prediction of eqn (4) is excellent within the measurement resolution, demonstrating that the magnetic 

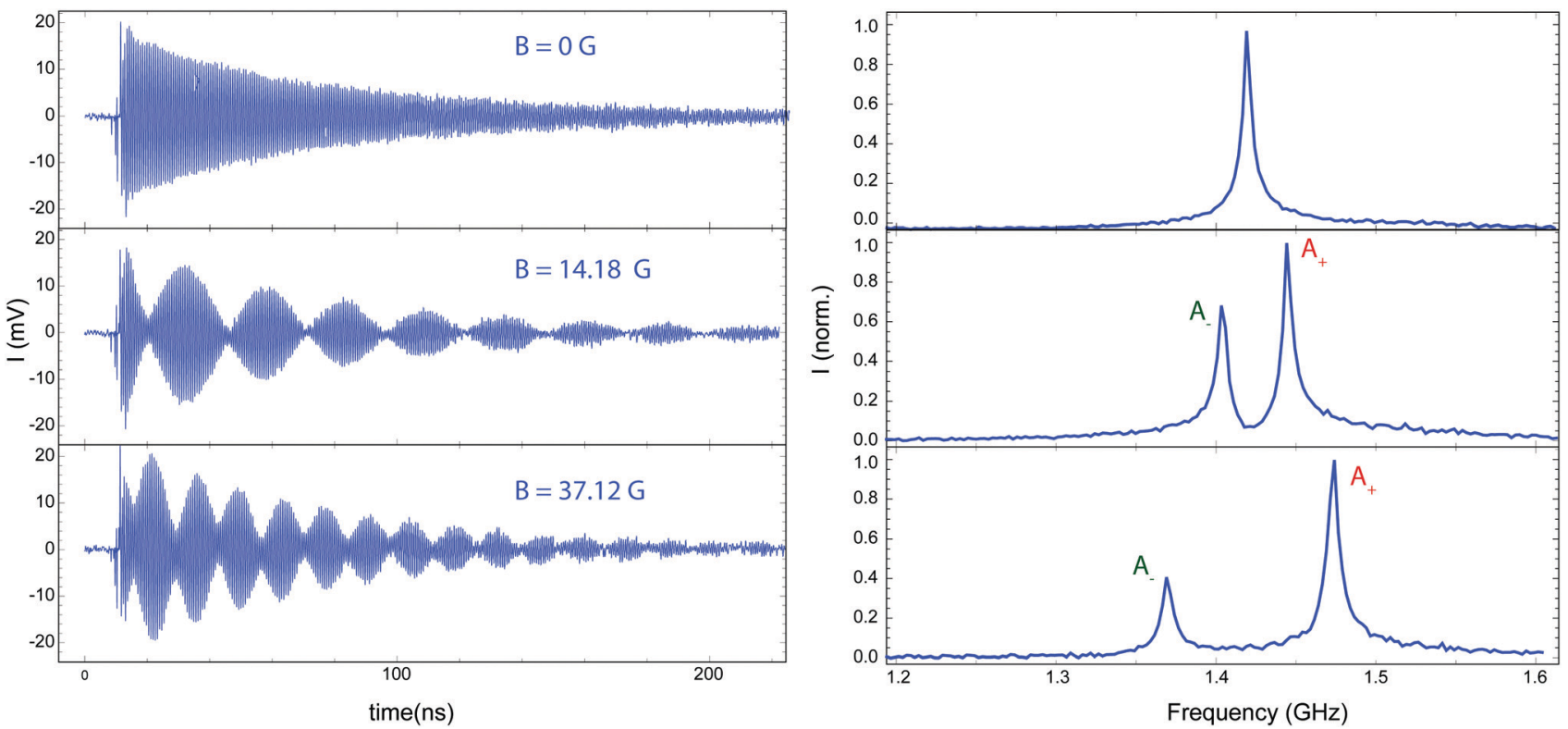

Fig. 2 Measured signal in the time domain (left) and the corresponding amplitude of Fourier transform (right) for different values of magnetic fields.

field can be estimated from the Fourier peaks. By repeating the measurement multiple times we estimate that the frequency statistical uncertainty (standard deviation) was around $40 \mathrm{kHz}$ corresponding to approximately $30 \mathrm{mG}$ sensitivity after 50 iterations. In this case, the sensitivity was limited by the poor resolution in Fourier transform, the electronic noise from an unoptimized electric circuitry and from discharge noise originating from pumping the photodissociating laser.

\section{Extension to time-dependent magnetometry}

The magnetometer that relies on the frequency content of Fourier power spectrum is not appropriate for characterizing a time-varying field. For such an estimation, the phase evolution of the signal should also be taken into account. In addition, high resolution detection requires first-order sensitivity to the magnetic field. The configuration that satisfies these conditions is shown in Fig. 1b: the dissociation/pumping axis, the magnetic field direction and the detection axis are all perpendicular to each other. With this scheme, the signal $\mathcal{E}$ (electromotive force in the detection coil) at time $t$ after the optical pumping can be approximated to be (see 7 for details):

$$
\mathcal{E}(t)=\mathcal{G} \omega_{0} \mathcal{N} \mu_{\mathrm{B}} N_{\mathrm{SPH}} \mathrm{e}^{-t / T_{2}} \sin \left[\gamma_{\mathrm{H}} \int_{0}^{t} B\left(t^{\prime}\right) \mathrm{d} t^{\prime}\right] \sin \left(\omega_{0} t\right)
$$

where $\mathcal{G}$ is a geometrical factor that relates the magnetic field from the spins to the flux in the region of the coil, $\mathcal{N}$ is the number of turns per unit length of the coil, $\mu_{\mathrm{B}}$ is the Bohr magneton, $N_{\mathrm{SPH}}$ the number of SPH atoms in the detection region, and $T_{2}$ is the hyperfine coherence time. The above equation is an approximation in the limit where the magnetic field evolves much slower compared to the hyperfine frequency and when $\omega_{0} \gg\left(1 / T_{2}, \gamma_{\mathrm{H}} B\right)$. It also assumes an inductor detection time constant much shorter than the hyperfine period and neglects dipolar-interactions between the hydrogen

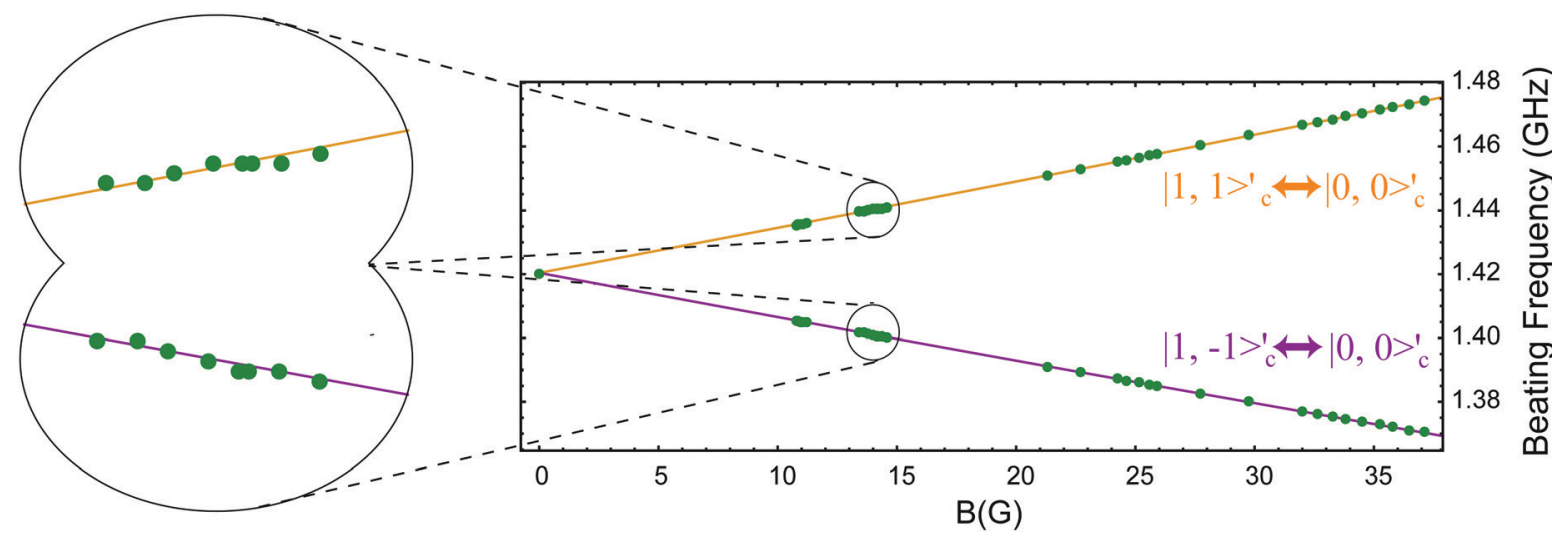

Fig. 3 Measured hyperfine frequencies versus $B$, and predictions of eqn (2) ( $\Delta f_{1 \sigma} \approx 40 \mathrm{kHz}$, smaller than the size of the points). 
spins. It can be seen that the information about the magnetic field appears as amplitude modulation of the carrier wave at the hyperfine frequency $\omega_{0}$. As a result, the evolution of magnetic fields at timescales longer than $1 / \omega_{0}$ should be faithfully captured with the proposed magnetometer as long as it is completed before significant spin-depolarization occurs.

\section{Simulations}

In Fig. 4 we show the results of simulations for time-varying magnetic waveforms: (a) is for cosine waves at $1 \mathrm{MHz}$ and $100 \mathrm{MHz}$, while (b) is for Gaussian magnetic pulses with durations (Gaussian RMS width) of 1 and $5 \mathrm{~ns}$, centered at 30 ns after the pumping pulse. The same magnetic field amplitude (maximum value) was considered for all the cases. In all the simulations the depolarization time was taken to be $0.75 \mu \mathrm{s}$. The magnetic field was estimated by first demodulating the signal at frequency $\omega_{0}$ and then taking the time derivative of the demodulation output. For the demodulation we implemented a simple low pass filter, where the signal was multiplied by $\cos \left(\omega_{0} t\right)$ and the resultant was integrated over a single period $2 \pi / f_{0}$. Subfigures (a) and (b) are plotted in the same (arbitrary) units for comparison. The response in the two cosine waves in (a) is similar despite a two orders of magnitude difference in frequencies. In (b) the retrieved Gaussian pulses (dotted blue lines) approximate well the actual Gaussian waveforms (red lines). In sub-figure (c), the response to a cosinusoidal magnetic field is plotted as a function of frequency. Here, the response is taken to be the maximum oscillation amplitude in the readout signal; in the examined frequency range, this maximum occurs at timescales much shorter than the decay time and is therefore not affected by the decay. The magnetometer bandwidth, defined as the frequency where the response has decreased by a factor of two, is approximately $450 \mathrm{MHz}$.

\section{Noise sources and sensitivity limit}

The detection of magnetic fields is fundamentally limited by the spin-projection noise, ${ }^{11}$ originating from the Heisenberg uncertainty for spins. Considering the experimental setup shown in Fig. 1b, the effect of spin noise on the magnetometer depends on the magnetic waveform. We consider a magnetic field of the form: $B(t)=B_{0} \mathcal{K}(t)$, where $B_{0}$ signifies the amplitude of magnetic field to be estimated from the magnetometer signal and $\mathcal{K}$ is an arbitrary function of time $t$ assumed to be known. By adopting a $\chi^{2}$ minimization, it can be shown (see 8) that the spin-projection noise limit for a single measurement run is given by:

$\delta B_{0}^{2}=\frac{2}{N_{\mathrm{SPH}}} \frac{\int_{0}^{T_{\mathrm{m}}} \int_{0}^{T_{\mathrm{m}}} \mathrm{d} t \mathrm{~d} t^{\prime} \mathrm{e}^{-\left(t+t^{\prime}\right) / T_{2}} \mathrm{e}^{-\left|t-t^{\prime}\right| / T_{2}} \int_{0}^{t} \mathcal{K}(x) \mathrm{d} x \int_{0}^{t^{\prime}} \mathcal{K}\left(x^{\prime}\right) \mathrm{d} x^{\prime}}{\left[\gamma_{\mathrm{H}} \int_{0}^{T_{\mathrm{m}}} \mathrm{d} t \mathrm{e}^{-2 t / T_{2}}\left(\int_{0}^{t} \mathcal{K}(x) \mathrm{d} x\right)^{2}\right]^{2}}$

where $T_{\mathrm{m}}$ is the measurement time after the photodissociation. For concreteness we consider the case of a cosinusoidal signal $(\mathcal{K}=\cos \omega t$, where $\omega$ is the angular frequency) and the conditions described above: SPH density $10^{18} \mathrm{~cm}^{-3}$, measurement volume $\left(100 \mu_{\mathrm{m}}\right)^{2} \times 1 \mathrm{~mm}=10 \mathrm{nl}$ and $T_{2} \approx 100 \mathrm{~ns}$. Such a projection-noise-limited magnetometer presents sensitivity on the order of a few nanoTesla after measurement time of 1 ns. For $T_{\mathrm{m}} \sim T_{2}$ the sensitivity is better than 200 pT per pulse; in this case, with a pulse repetition rate of $1 \mathrm{MHz}$, the projected sensitivity becomes better than $200 \mathrm{fT} / \sqrt{\mathrm{Hz}}$.

The magnetometer is also inflicted from thermal and quantum fluctuations in the detection coil and associated electric circuit. The impact of this noise source depends on the geometric arrangement of the spin-ensemble and the pick-up inductor. Furthermore, for non-spherical measurement volumes the dipolar interactions between the spins impair the magnetometer operation. We defer the consideration of these issues to a later study.

\section{Outlook}

The presented magnetometer is amenable for miniaturization. The measurement lengthscale, determined by the size of the photodissociation beam, can be readily adapted to the submicrometer region. Due to the high densities that can be achieved, the number of polarized atoms remains sufficient for high sensitivities even with such small measurement regions. Employing large spin-polarized density also relaxes the requirements on the induction coil. Unless high sensitivities are pursued or the measurement is performed from a very

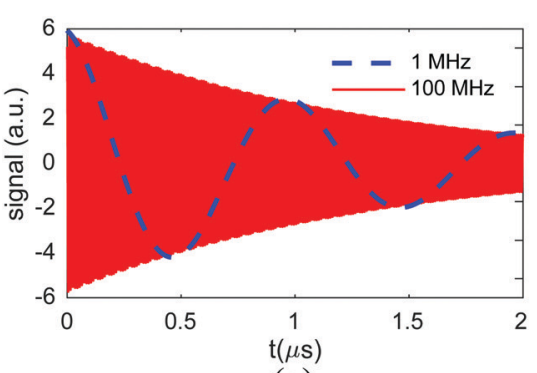

(a)

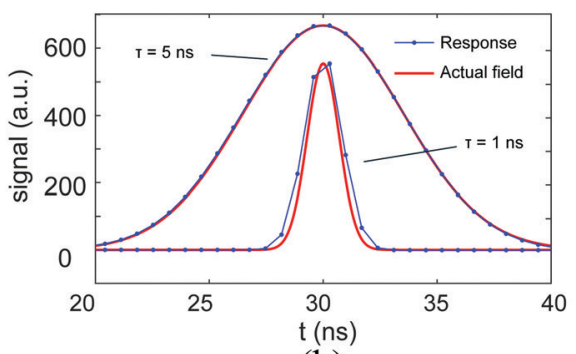

(b)

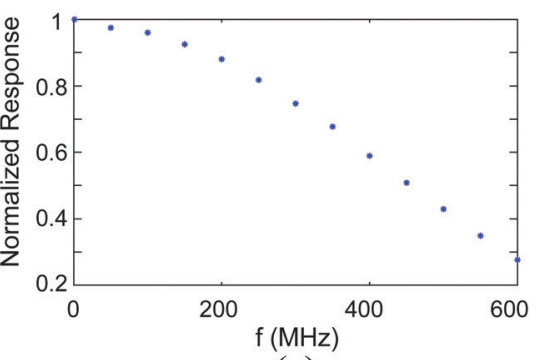

(c)

Fig. 4 Numerical simulation assuming atomic spin decay time $0.75 \mu \mathrm{s}$, for the detection geometry in Fig. $1 \mathrm{~b}$. (a) $B(t)=B_{0} \cos (2 \pi f t)$, where $B_{0}=100 \mu \mathrm{G}$ and $f=1 \mathrm{MHz}$ (blue dashed line) or $f=100 \mathrm{MHz}$ (red solid line). (b) $B(t)=B_{0} \mathrm{e}^{-\frac{\left(t-t_{0}\right)^{2}}{\Delta t^{2}}}$, where $\Delta t=1 \mathrm{~ns}$ and $5 \mathrm{~ns}, t_{0}=30 \mathrm{~ns}$ and $B_{0}=100 \mu \mathrm{G}$. (c) Frequency response of the magnetometer (normalized to DC response). 
small region, moderate coil quality factor and roomtemperature, standard electric circuitry should be adequate to detect the response of the polarized spins to the magnetic field. Another attractive feature is the large dynamic range and the capacity to operate in relatively high magnetic field backgrounds. The upper detection limit $B_{1}$ is set by the condition $\gamma_{\mathrm{H}} B_{1} \ll \omega_{0}$, which for SPH corresponds to $B_{1}$ in the hundreds of Gauss range.

Contrary to the conventional alkali-metal atomic magnetometers, the proposed scheme does not rely on a magnetic resonance condition. Therefore the detection bandwidth is not determined by the decoherence rate, but only from the hyperfine frequency. In the new scheme the spin-state preparation results from a single-step mechanism (photodissociation of the parent molecule) and does not involve several excitation cycles or the application of magnetic pulses to create coherences. Unlike magnetometry with nitrogen-vacancy centers in diamond, the dense spin ensemble does not suffer from inhomogeneous broadening and high sensitivities can be achieved without the use of dynamic decoupling techniques. This approach can be applied to different alkali-metal halides with larger hyperfine splittings. For instance, operation with $\mathrm{Cs}$ instead of $\mathrm{H}$ will extend the range of application to the sub-ns regime.

This fast and atomic magnetometer can be improved further in time resolution and detection sensitivity, by replacing the pickup coil with optical detection (via Faraday rotation), as in conventional atomic magnetometers. This is not practical for $\mathrm{H}$ atoms, for which there are only transitions in the VUV. However, polarized atomic photofragments with high atomic number can have convenient probe transitions in the UV, visible, or IR, and can have hyperfine beating frequencies of around $10 \mathrm{GHz}$ (e.g. Cs atoms), thus bringing fast magnetometry down to the 100 ps timescales. A magnetometer with such capabilities holds great promise for characterizing fast magnetization dynamics in chemical processes, including biologically relevant pathways, as well as in materials science and technology.

\section{Conflicts of interest}

There are no conflicts to declare.

\section{Appendix A: time evolution}

In the presence of a magnetic field $\mathbf{B}$ (which can be time varying), the SPH evolves according to the Hamiltonian (we neglect for the moment the relaxation):

$$
\hat{H}=\omega_{0} \hat{\mathbf{S}} \cdot \hat{\mathbf{I}}+\frac{g_{\mathrm{s}} \mu_{\mathrm{B}}}{\hbar} \mathbf{B} \cdot \hat{\mathbf{S}}=\hat{H}_{0}+\frac{g_{\mathrm{s}} \mu_{\mathrm{B}}}{\hbar} B \cdot \hat{S}_{z}
$$

where $\hat{\mathbf{I}}$ is the dimensionless nuclear spin operator, $\omega_{0}$ is the hyperfine frequency of $\mathrm{H}, \mu_{\mathrm{B}}$ is the Bohr magneton, $g_{\mathrm{s}} \approx 2$ is the electron spin $g$-factor, $\hat{H}_{0}$ is the Hamiltonian for hyperfine interaction expressed in (angular) frequency units. In the above equation, we neglected the coupling of the magnetic field to nuclear spin, as this is three orders of magnitude smaller than the coupling to the electron spin.
For clarity we write the Hamiltonian in the coupled (subscript c) and uncoupled (subscript $\mathrm{u}$ ) basis taking $z$ (the magnetic field direction) as the quantization axis:

$$
\begin{aligned}
\hat{H}_{\mathrm{u}}= & \left(\begin{array}{cccc}
\frac{\omega_{0}}{4}+\gamma_{\mathrm{H}} B & 0 & 0 & 0 \\
0 & -\frac{\omega_{0}}{4}-\gamma_{\mathrm{H}} B & \frac{\omega_{0}}{2} & 0 \\
0 & \frac{\omega_{0}}{2} & -\frac{\omega_{0}}{4}+\gamma_{\mathrm{H}} B & 0 \\
0 & 0 & 0 & \frac{\omega_{0}}{4}-\gamma_{\mathrm{H}} B
\end{array}\right) \\
\hat{H}_{\mathrm{c}}= & \left(\begin{array}{cccc}
\frac{\omega_{0}}{4}+\gamma_{\mathrm{H}} B & 0 & 0 & 0 \\
0 & \frac{\omega_{0}}{4} & 0 & \gamma_{\mathrm{H}} B \\
0 & 0 & \frac{\omega_{0}}{4}-\gamma_{\mathrm{H}} B & 0 \\
0 & \gamma_{\mathrm{H}} B & 0 & -\frac{3 \omega_{0}}{4}
\end{array}\right),
\end{aligned}
$$

where $\gamma_{\mathrm{H}}=g_{\mathrm{S}} \mu_{\mathrm{B}} / 2 \hbar$ is the gyromagnetic ratio of atomic $\mathrm{H}$. The above matrices are expressed in a basis with the following ordering:

$$
\begin{array}{ll}
\text { Uncoupled basis: } & \left\{\left|m_{\mathrm{s}}=1 / 2, m_{\mathrm{I}}=1 / 2\right\rangle,\right. \\
& \left|m_{\mathrm{s}}=-1 / 2, m_{\mathrm{I}}=1 / 2\right\rangle, \\
& \left|m_{\mathrm{s}}=1 / 2, m_{\mathrm{I}}=-1 / 2\right\rangle, \\
& \left.\left|m_{\mathrm{s}}=-1 / 2, m_{\mathrm{I}}=-1 / 2\right\rangle\right\}, \\
\text { Coupled basis: } \quad & \left\{\left|F=1, m_{\mathrm{F}}=1\right\rangle,\right. \\
& \left|F=1, m_{\mathrm{F}}=0\right\rangle, \\
& \left|F=1, m_{\mathrm{F}}=-1\right\rangle, \\
& \left.\left|F=0, m_{\mathrm{F}}=0\right\rangle\right\},
\end{array}
$$

where $F$ is the total spin (sum of electronic and nuclear spin) quantum number, $m_{\mathrm{s}}, m_{\mathrm{I}}$ and $m_{\mathrm{F}}$ are respectively the electronic, nuclear and total spin projection along the quantization axis.

Transformation of an arbitrary operator $\hat{\mathcal{O}}$ or state vector $|\psi\rangle$ from one basis to the other can be performed according to the following rules:

$$
\begin{aligned}
\hat{\mathcal{O}}_{\mathrm{c}} & =\mathcal{T}_{\mathrm{cu}} \cdot \hat{\mathcal{O}}_{\mathrm{u}} \cdot \mathcal{T}_{\mathrm{cu}}{ }^{-1}, \\
\hat{\mathcal{O}}_{\mathrm{u}} & =\mathcal{T}_{\mathrm{cu}}{ }^{-1} \cdot \hat{\mathcal{O}}_{\mathrm{c}} \cdot \mathcal{T}_{\mathrm{cu}}, \\
|\psi\rangle_{\mathrm{c}} & =\mathcal{T}_{\mathrm{cu}}|\psi\rangle_{\mathrm{u}} \\
|\psi\rangle_{\mathrm{u}} & =\mathcal{T}_{\mathrm{cu}}{ }^{-1}|\psi\rangle_{\mathrm{c}}
\end{aligned}
$$




$$
\mathcal{T}_{\mathrm{cu}}=\left(\begin{array}{cccc}
1 & 0 & 0 & 0 \\
0 & \frac{1}{\sqrt{2}} & \frac{1}{\sqrt{2}} & 0 \\
0 & 0 & 0 & 1 \\
0 & -\frac{1}{\sqrt{2}} & \frac{1}{\sqrt{2}} & 0
\end{array}\right)
$$

The hydrohalide photo-dissociation occurs with a subnanosecond laser pulse and optical pumping effectively transfers angular momentum from light to the electronic spin, leaving the nuclear spin degrees of freedom in their thermal (completely unpolarized) state. Following the above basis ordering, after optical pumping half of the polarized $\mathrm{H}$ atoms are in the quantum state (expressed as a column vector): where $|\psi(t)\rangle$ is the wavefunction at time $t$ after optical pumping. For $|\psi(0)\rangle=|\psi(0)\rangle_{1}$ the wavefunction at time $t$ in the coupled basis is: where: $\tilde{\omega}=\sqrt{\omega_{0}^{2}+4 \gamma_{\mathrm{H}}^{2} B^{2}}$.

In the general case of a time varying magnetic field the Schrödinger equation cannot be solved analytically, as the Hamiltonian does not commute with itself at different times. For an approximate analytical solution it is convenient to work in the interaction picture (denoted by the $\sim$ symbol):

$$
\frac{\partial}{\partial t}|\tilde{\psi}(t)\rangle=-\imath \hat{\tilde{V}}|\tilde{\psi}(t)\rangle
$$

where $\hat{\tilde{V}}$ is the Hamiltonian describing the magnetic field coupling to the atoms:

$$
|\psi(0)\rangle_{0}=\left|\psi_{0}\right\rangle=\left\{\begin{array}{l}
(1,0,0,0)_{\mathrm{u}, y}^{\mathbf{T}} \stackrel{\hat{\mathcal{R}}_{x} \cdot(1,0,0,0)^{\mathbf{T}}}{\longrightarrow}\left(\frac{1}{2},-\frac{l}{2},-\frac{l}{2},-\frac{1}{2}\right)_{\mathrm{u}}^{\mathbf{T}} \stackrel{\mathcal{T}_{\mathrm{cu}}}{\longrightarrow}\left(\frac{1}{2},-\frac{l}{2},-\frac{1}{2}, 0\right)_{\mathrm{c}}^{\mathbf{T}}, \quad \sigma=1 \\
(0,0,0,1)_{\mathrm{u}, y}^{\mathbf{T}} \stackrel{\hat{\mathcal{R}}_{x} \cdot(0,0,0,1)^{\mathbf{T}}}{\longrightarrow}\left(-\frac{1}{2},-\frac{l}{2},-\frac{l}{2}, \frac{1}{2}\right)_{\mathrm{u}}^{\mathbf{T}} \stackrel{\mathcal{T}_{\mathrm{cu}}}{\longrightarrow}\left(-\frac{1}{2},-\frac{l}{2}, \frac{1}{2}, 0\right)_{\mathrm{c}}^{\mathbf{T}}, \quad \sigma=-1
\end{array},\right.
$$

where the subscript $y$ denotes that the quantization axis for spin projections was taken in the $y$ direction (if no axis subscript appears it is implicitly assumed that the quantization axis is in the $z$ direction), $\mathbf{T}$ is the transpose operation, $\sigma$ is the helicity of the pumping light pulse and $\hat{\mathcal{R}}_{x}$ is the rotation matrix around the $x$-axis applied to the uncoupled basis (two spins, each of spin 1/2):

$$
\hat{\mathcal{R}}_{x}=\mathrm{e}^{-l \hat{S}_{x} \frac{\pi}{2}} \otimes \mathrm{e}^{-l \hat{S}_{x} \frac{\pi}{2}} .
$$

$$
\begin{aligned}
\hat{\tilde{V}}(t) & =\mathrm{e}^{l \hat{H}_{0} t}\left[g_{\mathrm{s}} \mu_{\mathrm{B}} B \hat{S}_{z}\right] \mathrm{e}^{-l \hat{H}_{0} t} \\
& =\gamma_{\mathrm{H}} B(t)\left(\begin{array}{cccc}
1 & 0 & 0 & 0 \\
0 & -\cos \left(\omega_{0} t\right) & \imath \sin \left(\omega_{0} t\right) & 0 \\
0 & -l \sin \left(\omega_{0} t\right) & \cos \left(\omega_{0} t\right) & 0 \\
0 & 0 & 0 & -1
\end{array}\right) .
\end{aligned}
$$

The other half of the polarized $\mathrm{H}$ atoms are in the state:

$$
|\psi(0)\rangle_{1}=\left|\psi_{1}\right\rangle=\left\{\begin{array}{l}
(0,0,1,0)_{\mathrm{u}, y}^{\mathbf{T}} \stackrel{\hat{\mathcal{R}}_{x} \cdot(0,0,1,0)^{\mathbf{T}}}{\longrightarrow}\left(-\frac{l}{2},-\frac{1}{2}, \frac{1}{2},-\frac{l}{2}\right)_{\mathrm{u}}^{\mathbf{T}} \stackrel{\mathcal{T}_{\mathrm{cu}}}{\longrightarrow}\left(-\frac{l}{2}, 0,-\frac{l}{2}, \frac{1}{\sqrt{2}}\right)_{\mathrm{c}}^{\mathbf{T}}, \quad \sigma=1 \\
(0,1,0,0)_{\mathrm{u}, y}^{\mathbf{T}} \stackrel{\hat{\mathcal{R}}_{x} \cdot(0,1,0,0)^{\mathbf{T}}}{\longrightarrow}\left(-\frac{l}{2}, \frac{1}{2},-\frac{1}{2},-\frac{l}{2}\right)_{\mathrm{u}}^{\mathbf{T}} \stackrel{\mathcal{T}_{\mathrm{cu}}}{\longrightarrow}\left(-\frac{l}{2}, 0,-\frac{l}{2},-\frac{1}{\sqrt{2}}\right)_{\mathrm{c}}^{\mathbf{T}}, \quad \sigma=-1
\end{array} .\right.
$$

For the observable $\frac{\mathrm{d} \hat{S}_{x}}{\mathrm{~d} t}$ the contribution to signal of atoms initially at state $|\psi(0)\rangle_{0}$ is a factor of $\gamma_{\mathrm{H}} B / \omega_{0}$ smaller than the contribution of atoms initially at $|\psi(0)\rangle_{1}$. In the case of $\gamma_{\mathrm{H}} B / \omega_{0} \ll 1$, the magnetometer signal is mainly determined by the atoms initially at $|\psi(0)\rangle_{1}$.

For static magnetic field, Schrödinger equation can be solved in a straightforward manner:

$$
\begin{aligned}
l \frac{\partial}{\partial t}|\psi(t)\rangle & =\hat{H}|\psi(t)\rangle \Rightarrow|\psi(t)\rangle=\mathrm{e}^{-l \hat{H} t}|\psi(0)\rangle \quad(16) \\
|\psi(t)\rangle_{1} & =\left(-\frac{1}{2} l \mathrm{e}^{-l\left(\frac{\omega_{0}}{4}+\gamma_{\mathrm{H}} B\right) t},-l \sigma \frac{\sqrt{2} \gamma_{\mathrm{H}} B \mathrm{e}^{l \frac{\omega_{0}}{4} t} \sin \left[\frac{1}{2} \tilde{\omega} t\right]}{\tilde{\omega}},-\frac{1}{2} l \mathrm{e}^{-l\left(\frac{\omega_{0}}{4}-\gamma_{\mathrm{H}} B\right) t}, \sigma \frac{\mathrm{e}^{l \frac{\omega_{0}}{4} t}\left(l \omega_{0} \sin \left[\frac{1}{2} \tilde{\omega} t\right]+\tilde{\omega} \cos \left[\frac{1}{2} \tilde{\omega} t\right]\right)}{\sqrt{2} \tilde{\omega}}\right) \\
& \approx-\frac{1}{2} l \mathrm{e}^{l \frac{1}{4}\left(\omega_{0}+2 \tilde{\omega}\right) t}\left(\mathrm{e}^{-l\left(\frac{\omega_{0}}{2}+\gamma_{\mathrm{H}} B+\frac{1}{2} \tilde{\omega}\right) t}, 0, \mathrm{e}^{-l\left(\frac{\omega_{0}}{2}-\gamma_{\mathrm{H}} B+\frac{1}{2} \tilde{\omega}\right) t}, l \sqrt{2} \sigma\right)^{\mathbf{T}}
\end{aligned}
$$

In the last equation $\hat{\tilde{V}}$ is expressed in the uncoupled basis.

The solution of eqn (18) can be expressed in the form of the Magnus series. ${ }^{25}$ For magnetic fields that change slowly with respect to the hyperfine frequency so that:

$$
\int_{0}^{t} B\left(t^{\prime}\right) \mathrm{e}^{l \omega_{0} t^{\prime}} \mathrm{d} t^{\prime} \ll \int_{0}^{t} B\left(t^{\prime}\right) \mathrm{d} t^{\prime}
$$


the solution of eqn (18) can be approximated by keeping only the first term in Magnus expansion. In this case:

$$
|\tilde{\psi}(t)\rangle \approx \exp \left(-l \int_{0}^{t} \hat{\tilde{V}}\left(t^{\prime}\right) \mathrm{d} t^{\prime}\right)|\psi(0)\rangle
$$

and the observable (taking into account the condition (20)) can be written as:

$$
\begin{aligned}
& \frac{\mathrm{d}}{\mathrm{d} t}\left\langle\tilde{\psi}(t)\left|\mathrm{e}^{i \hat{H}_{0} t} \hat{S}_{x} \mathrm{e}^{-l \hat{H}_{0} t}\right| \tilde{\psi}(t)\right\rangle \\
& \approx \sigma \frac{\mathrm{d}}{\mathrm{d} t} \times\left\{\frac{1}{2} \sin \left[\gamma_{\mathrm{H}} \int_{0}^{t} B\left(t^{\prime}\right) \mathrm{d} t^{\prime}\right] \cos \left(\omega_{0} t\right)\right\} \\
& \quad \approx-\sigma \frac{\omega_{0}}{2} \times \sin \left[\gamma_{\mathrm{H}} \int_{0}^{t} B\left(t^{\prime}\right) \mathrm{d} t^{\prime}\right] \sin \left(\omega_{0} t\right) .
\end{aligned}
$$

The last approximation holds for $\gamma_{\mathrm{H}} B \ll \omega_{0}$.

The condition stated in 20 implies that the magnetic field does not induce hyperfine transitions and the magnetic field can be treated as a perturbation to the energies of the hyperfine levels. Then, for small magnetic fields $\left(\gamma_{\mathrm{H}} B \ll \omega_{0}\right)$ the Hamiltonian for $\mathrm{H}$ atoms (hyperfine levels with $F=0$ and $F=1$ ) can be approximated to be (as before the magnetic field direction is taken to be the quantization axis):

$$
\hat{H} \approx \hat{H}_{0}+\gamma_{\mathrm{H}} B(t)\left(\hat{S}_{z}+\hat{I}_{z}\right)
$$

This Hamiltonian commutes with itself at different times and the Schrödinger equation can be solved analytically:

$$
|\psi(t)\rangle=\mathrm{e}^{-l \int_{0}^{t} \hat{H}\left(t^{\prime}\right) \mathrm{d} t^{\prime}}|\psi(0)\rangle
$$

\section{Decay}

The evolution of spins is also affected by relaxation processes leading to non-Hamiltonian dynamics. We model these by introducing a decay term in the density matrix equation:

$$
\frac{\mathrm{d} \rho}{\mathrm{d} t}=\imath[\rho, H]-\frac{1}{T_{2}}\left(\rho-\rho_{\mathrm{eq}}\right),
$$

where $1 / T_{2}$ is the decay rate, and $\rho_{\text {eq }}$ corresponds to the state towards which the decay processes drive the system. We take this quantum state to be the completely unpolarized state, written in the form:

$$
\rho_{\text {eq }}=\frac{1}{4} \rrbracket_{4 \times 4},
$$

where $\mathbb{q}_{4 \times 4}$ is the $4 \times 4$ identity matrix. For eqn (25) and (26) to be valid the population decay (hydrogen atom losses due to formation of molecules) should be significantly slower compared to (atomic) hydrogen spin-decoherence processes. This condition is justified in our case.
The density matrix at $t=0$ is ( $z$ quantization axis the direction of magnetic field and optical pumping in the $y$ axis):

$$
\rho(0)=\frac{1}{2}\left|\psi_{0}\right\rangle\left\langle\psi_{0}\left|+\frac{1}{2}\right| \psi_{1}\right\rangle\left\langle\psi_{1}\right| \rightarrow\left(\begin{array}{ccccc}
\frac{1}{4} & \sigma \frac{l}{4} & 0 & 0 \\
-\sigma \frac{l}{4} & \frac{1}{4} & 0 & 0 \\
0 & 0 & \frac{1}{4} & \sigma \frac{l}{4} \\
0 & 0 & -\sigma \frac{l}{4} & \frac{1}{4}
\end{array}\right)_{\mathrm{u}}
$$

An analytical solution to the density matrix equation can be found for a static magnetic field or for an arbitrary field when the Hamiltonian can be approximated by eqn (23). In the experimentally relevant limit of $\omega_{0} \gg\left(1 / T_{2}, \gamma_{\mathrm{H}} B\right)$ the signal is (keeping lowest order terms in the harmonic amplitudes):

$$
\begin{aligned}
\left\langle\frac{\mathrm{d} \hat{S}_{x}}{\mathrm{~d} t}\right\rangle(t)= & \operatorname{Tr}\left[\frac{\mathrm{d} \rho}{\mathrm{d} t} \hat{S}_{x}\right] \approx-\sigma \frac{1}{4} \mathrm{e}^{-t / T_{2}} \\
& \times \sin \left[\gamma_{\mathrm{H}} \int_{0}^{t} B\left(t^{\prime}\right) \mathrm{d} t^{\prime}\right] \sin \left(\omega_{0} t\right) .
\end{aligned}
$$

Eqn (25) and (26) implies spin-damping occurs (at equal rate) for both electron and nuclear spin of $\mathrm{H}$. However, the results derived here are general for the relevant approximations $\left(\omega_{0} \gg\left(1 / T_{2}, \gamma_{\mathbf{H}} B\right)\right)$ and condition (20). For instance, eqn (28) is reproduced also for decay mechanisms that relax only the electronic spin:

$$
\frac{\mathrm{d} \rho}{\mathrm{d} t}=\imath[\rho, H]-\frac{4}{3 T_{2}}\left(\hat{\mathbf{S}}^{2} \cdot \rho-\hat{\mathbf{S}} \cdot \rho \hat{\mathbf{S}}\right) .
$$

\section{Appendix B: spin-projection noise}

In the following we will need to know the multi-time correlation

$$
\left\langle\frac{\mathrm{d} \hat{S}_{x}}{\mathrm{~d} t}(t) \frac{\mathrm{d} \hat{S}_{x}}{\mathrm{~d} t}\left(t^{\prime}\right)\right\rangle,
$$

where we take

$$
\frac{\mathrm{d}}{\mathrm{d} t} \hat{S}_{x}=\imath\left[\hat{H}, \hat{S}_{x}\right]-\frac{1}{T_{2}} \hat{S}_{x}
$$

The last term (not derived from first principles) was introduced to account for the spin decay. We assume that the evolution of 
the density matrix $\rho$ is given by eqn (25) (though the results are the same - within the relevant approximations - for the evolution described in eqn (29)).

The multi-time correlation can be written operationally in the form:

$$
\left\langle\frac{\mathrm{d} \hat{S}_{x}}{\mathrm{~d} t}(t) \frac{\mathrm{d} \hat{S}_{x}}{\mathrm{~d} t}\left(t^{\prime}\right)\right\rangle=\operatorname{Tr}\left\{\frac{\mathrm{d} \hat{S}_{x}}{\mathrm{~d} t}\left[\hat{U}\left(t, t^{\prime}\right)\left(\frac{\mathrm{d} \hat{S}_{x}}{\mathrm{~d} t} \rho\left(t^{\prime}\right)\right)\right]\right\},
$$

where $U\left(t, t^{\prime}\right)$ is the evolution operator of the density matrix from time $t^{\prime}$ to $t\left(t>t^{\prime}\right)$, and $\rho\left(t^{\prime}\right)$ is the density matrix at time $t^{\prime}$ : $\rho\left(t^{\prime}\right)=U\left(t^{\prime}, 0\right) \rho(0)$. The evolution operator $U$ cannot be written in the form of a matrix and is not associative. The evolved state $\hat{U}\left(t, t^{\prime}\right)\left(\frac{\mathrm{d} \hat{S}_{x}}{\mathrm{~d} t} \rho\left(t^{\prime}\right)\right)$ can be found from the general solution of eqn (25) taking $\frac{\mathrm{d} \hat{S}_{x}}{\mathrm{~d} t} \rho\left(t^{\prime}\right)$ as the initial condition for the density matrix. For quantum noise analysis we can take the magnetic field to be zero in the calculation of the multi-time correlation, since quantum noise affects considerably magnetometry only at low fields. When $\omega_{0} T_{2} \gg 1$, it can be found that:

$$
\left\langle\frac{\mathrm{d} \hat{S}_{x}}{\mathrm{~d} t}(t) \frac{\mathrm{d} \hat{S}_{x}}{\mathrm{~d} t}\left(t^{\prime}\right)\right\rangle=\frac{1}{8} \omega^{2} \mathrm{e}^{-\left|t-t^{\prime}\right| / T_{2}} \cos \left[\omega_{0}\left(t-t^{\prime}\right)\right] .
$$

In order to find what is the magnetic field uncertainty due to spin-projection noise, we have to specify a method for estimating the magnetic field from the detected signal $\frac{\mathrm{d} \hat{S}_{x}}{\mathrm{~d} t}(t)$. Taking into account eqn (28), one way to do this (appropriate for arbitrary magnetic waveforms) is from considering the "quasi-instantaneous" amplitude of the frequency component at the hyperfine frequency:

$$
\xi\left(n T_{\mathrm{hf}}\right)=\frac{1}{T_{\mathrm{hf}}} \int_{n T_{\mathrm{hf}}}^{(n+1) T_{\mathrm{hf}}} \frac{\mathrm{d} \hat{S}_{x}}{\mathrm{~d} t}(t) \sin \left(\omega_{0} t\right) \mathrm{d} t,
$$

where $T_{\mathrm{hf}}=2 \pi / \omega_{0}$ is the period of hyperfine oscillation and $n$ is an integer number. We assume that the magnetic field can be written in the form: $B(t)=B_{0} \mathcal{K}(t)$, where $\mathcal{K}$ is a known (but other than this an arbitrary), time-dependent function. Spinprojection noise creates an uncertainty in the estimation of $B_{0}$.

We consider the case where the functions $\mathcal{K}(t)$ and $\mathrm{e}^{-t / T_{2}}$ evolve in time much slower compared to $\sin \left(\omega_{0} t\right)$ and can therefore be considered constant during the hyperfine period. Effectively this is the situation for $\omega_{0} T_{2} \gg 1$ and the condition stated in 20. Then, the quasi-amplitude of the sine wave at hyperfine frequency is (we ignore the - irrelevant for noise purposes $--\sigma$ factor in the signal in eqn (28)):

$$
\begin{aligned}
\frac{1}{T_{\mathrm{hf}}} \int_{n T_{\mathrm{hf}}}^{(n+1) T_{\mathrm{hf}}} \frac{\mathrm{d} \hat{S}_{x}}{\mathrm{~d} t}(t) \sin \left(\omega_{0} t\right) \mathrm{d} t & \approx \frac{1}{8} \omega_{0} \mathrm{e}^{-n T_{\mathrm{hf}} / T_{2}} \sin \left[\gamma_{\mathrm{H}} B_{0} \int_{0}^{n T_{\mathrm{hf}}} \mathcal{K}\left(t^{\prime}\right) \mathrm{d} t^{\prime}\right] \\
& \approx \frac{1}{8} \omega_{0} \mathrm{e}^{-n T_{\mathrm{hf}} / T_{2}} \gamma_{\mathrm{H}} B_{0} \int_{0}^{n T_{\mathrm{hf}}} \mathcal{K}\left(t^{\prime}\right) \mathrm{d} t^{\prime},
\end{aligned}
$$

where the last approximation holds for small magnetic fields.
The magnetic field $B_{0}$ can be estimated by minimizing with respect to the parameter $B_{0}$ the $\chi^{2}$ function:

$$
\chi^{2}=\sum_{n=0}^{M}\left[\frac{1}{8} \omega_{0} \mathrm{e}^{-n T_{\mathrm{hf}} / T_{2}} \gamma_{\mathrm{H}} B_{0} \int_{0}^{n T_{\mathrm{hf}}} \mathcal{K}\left(t^{\prime}\right) \mathrm{d} t^{\prime}-\xi\left(n T_{\mathrm{hf}}\right)\right]^{2},
$$

where $\mathrm{MT}_{\mathrm{hf}}$ is the total measurement time. The above equation can be viewed as a curve fitting problem with unknown parameter $B_{0}$ for a noisy signal. The solution to the curve fitting problem is:

$$
B_{0}=\frac{\sum_{n=0}^{M} \xi\left(n T_{\mathrm{hf}}\right) \mathrm{e}^{-n T_{\mathrm{hf}} / T_{2}} \int_{0}^{n T_{\mathrm{hf}}} \mathcal{K}\left(t^{\prime}\right) \mathrm{d} t^{\prime}}{\gamma_{\mathrm{H}} \sum_{n=0}^{K} \frac{1}{8} \omega_{0} \mathrm{e}^{-2 n T_{\mathrm{hf}} / T_{2}}\left(\int_{0}^{n T_{\mathrm{hf}}} \mathcal{K}\left(t^{\prime}\right) \mathrm{d} t^{\prime}\right)^{2}}
$$

and the estimation uncertainty in $B_{0}$ due to the spin-projection noise is:

$$
\begin{aligned}
& \delta B_{0}^{2}= \\
& \frac{\sum_{n^{\prime}=0}^{M} \sum_{n=0}^{M}\left\langle\xi\left(n T_{\mathrm{hf}}\right) \xi\left(n^{\prime} T_{\mathrm{hf}}\right)\right\rangle \mathrm{e}^{-\left(n+n^{\prime}\right) T_{\mathrm{hf}} / T_{2}} \int_{0}^{n T_{\mathrm{hf}}} \mathcal{K}\left(t^{\prime}\right) \mathrm{d} t^{\prime} \int_{0}^{n^{\prime} T_{\mathrm{hf}}} \mathcal{K}\left(t^{\prime \prime}\right) \mathrm{d} t^{\prime \prime}}{\gamma_{\mathrm{H}}^{2}\left[\sum_{n=0}^{K} \frac{1}{8} \omega_{0} \mathrm{e}^{-2 n T_{\mathrm{hf}} / T_{2}}\left(\int_{0}^{n T_{\mathrm{hf}}} \mathcal{K}\left(t^{\prime}\right) \mathrm{d} t^{\prime}\right)^{2}\right]^{2}} .
\end{aligned}
$$

From eqn (33) and (34) we find (in the limit of $\omega_{0} \gg \gamma$ ):

$$
\left\langle\xi\left(n T_{\mathrm{hf}}\right) \xi\left(n^{\prime} T_{\mathrm{hf}}\right)\right\rangle=\frac{1}{32} \omega_{0}^{2} \mathrm{e}^{-\left|\left(n-n^{\prime}\right)\right| T_{\mathrm{hf}} / T_{2}},
$$

So that:

$$
\delta B_{0}{ }^{2}=2 \frac{\int_{0}^{T_{\mathrm{m}}} \int_{0}^{T_{\mathrm{m}}} \mathrm{d} t \mathrm{~d} t^{\prime} \mathrm{e}^{-\left(t+t^{\prime}\right) / T_{2}} \mathrm{e}^{-\left|t-t^{\prime}\right| / T_{2}} \int_{0}^{t} \mathcal{K}\left(x^{\prime}\right) \mathrm{d} x^{\prime} \int_{0}^{t^{\prime}} \mathcal{K}(x) \mathrm{d} x}{\left[\gamma_{\mathrm{H}} \int_{0}^{T_{\mathrm{m}}} \mathrm{d} t \mathrm{e}^{-2 t / T_{2}}\left(\int_{0}^{t} \mathcal{K}(x) \mathrm{d} x\right)^{2}\right]^{2}},
$$

where $T_{\mathrm{m}}$ is the measurement time for a single run of the experiment.

The above derivation applies to a measurement a single $\mathrm{H}$ atom. For $N_{\mathrm{SPH}}$ independent (absence of spin-squeezing) $\mathrm{H}$ atoms the uncertainty in magnetic field estimation is:

$\delta B_{0}{ }^{2}=\frac{2}{N_{\mathrm{SPH}}} \frac{\int_{0}^{T_{\mathrm{m}}} \int_{0}^{T_{\mathrm{m}}} \mathrm{d} t \mathrm{~d} t^{\prime} \mathrm{e}^{-\left(t+t^{\prime}\right) / T_{2}} \mathrm{e}^{-\left|t-t^{\prime}\right| / T_{2}} \int_{0}^{t} \mathcal{K}\left(x^{\prime}\right) \mathrm{d} x^{\prime} \int_{0}^{t^{\prime}} \mathcal{K}(x) \mathrm{d} x}{\left[\gamma_{\mathrm{H}} \int_{0}^{T_{\mathrm{m}}} \mathrm{d} t \mathrm{e}^{-2 t / T_{2}}\left(\int_{0}^{t} \mathcal{K}(x) \mathrm{d} x\right)^{2}\right]^{2}}$

\section{Acknowledgements}

This work was supported by the Hellenic Foundation for Research and Innovation (HFRI) and the General Secretariat for Research and Technology (GSRT), grant agreement no. HFRI-FM17-3709 (project NUPOL) and by the project "HELLAS-CH" (MIS 5002735), which is implemented under the "Action for Strengthening Research and Innovation Infrastructures", funded by the Operational Programme "Competitiveness, Entrepreneurship and Innovation” (NSRF 2014-2020) 
and cofinanced by Greece and the European Union (European Regional Development Fund). GEK acknowledges funding from the Hellenic Foundation for Research and Innovation (HFRI) and the General Secretariat for Research and Technology (GSRT), under grant agreement No. [1789], and GV acknowledges funding from EU QuantERA Project PACE-IN (GSRT Grant No. T11EPA4-00015).

\section{References}

1 P. Ripka, Magnetic Sensors and Magnetometers, Artech House Publishers, 2001, ISBN 1580530575, 9781580530576.

2 R. Bradley, J. Clarke, D. Kinion, L. J. Rosenberg, K. van Bibber, S. Matsuki, M. Mück and P. Sikivie, Microwave cavity searches for dark-matter axions, Rev. Mod. Phys., 2003, 75, 777-817, DOI: 10.1103/RevModPhys. 75.777 .

3 O. I. Gnezdilov, A. E. Mambetov, A. A. Obynochny and K. M. Salikhov, Time-resolved epr study of electron spin polarization and spin exchange in mixed solutions of porphyrin stable free radicals, Appl. Magn. Reson., 2003, 250(1), 157, DOI: 10.1007/BF03166974, ISSN 16137507.

4 M. Mompeán, R. M. Sánchez-Donoso, A. de la Hoz, V. Saggiomo, A. H. Velders and M. Victoria Gomez, Pushing nuclear magnetic resonance sensitivity limits with microfluidics and photo-chemically induced dynamic nuclear polarization, Nat. Commun., 2018, 90(1), 108, DOI: 10.1038/s41467-017-02575-0, ISSN 2041-1723.

5 O. B. Morozova, K. L. Ivanov, A. S. Kiryutin, R. Z. Sagdeev, T. Köchling, H. M. Vieth and A. V. Yurkovskaya, Timeresolved CIDNP: An NMR way to determine the EPR parameters of elusive radicals, Phys. Chem. Chem. Phys., 2011, 130(14), 6619-6627.

6 J. Klein-Seetharaman, M. Oikawa, S. B. Grimshaw, J. Wirmer, E. Duchardt, T. Ueda, T. Imoto, L. J. Smith, C. M. Dobson and H. Schwalbe, Long-range interactions within a nonnative protein, Science, 2002, 2950(5560), 1719-1722, DOI: 10.1126/ science.1067680, ISSN 0036-8075.

7 Y. Acremann, C. H. Back, M. Buess, O. Portmann, A. Vaterlaus, D. Pescia and H. Melchior, Imaging precessional motion of the magnetization vector, Science, 2000, 290, 491-495, DOI: 10.1126/science.290.5491.492.

8 D. Atkinson, D. A. Allwood, M. D. Cooke and R. P. Cowburn. Nanosecond pulsed field magnetization reversal in thin-film permalloy studied by kerr effect magnetometry, in 2002 IEEE International Magnetics Conference (INTERMAG), 2002, p. GV4, DOI: 10.1109/INTMAG.2002.1001512.

9 M. Yamada, R. Kulsrud and H. Ji, Magnetic reconnection, Rev. Mod. Phys., 2010, 82, 603-664, DOI: 10.1103/RevModPhys. 82.603.

10 High Sensitivity Magnetometers. Smart Sensors, Measurement and Instrumentation 19, ed. S. C. Mukhopadhyay, A. Grosz and M. J. Haji-Sheikh, Springer International Publishing, 1st edn, 2017, ISBN 978-3-319-34070-8, 978-3-319-34068-5, https://books.google.es/books?id=t8gcDQAAQBAJLink.

11 D. Budker and M. Romalis, Optical magnetometry, Nat. Phys., 2007, 3, 227-234, DOI: 10.1038/nphys566.

12 R. Jiménez-Martínez, W. C. Griffith, S. Knappe, J. Kitching and M. Prouty, High-bandwidth optical magnetometer, J. Opt. Soc. Am. B, 2012, 290(12), 3398-3403, DOI: 10.1364/ JOSAB.29.003398.

13 R. L. Fagaly, Superconducting quantum interference device instruments and applications, Rev. Sci. Instrum., 2006, 770(10), 101101, DOI: 10.1063/1.2354545.

14 A. Bienfait, J. J. Pla, Y. Kubo, M. Stern, X. Zhou, C. C. Lo, C. D. Weis, T. Schenkel, M. L. W. Thewalt, D. Vion, D. Esteve, B. Julsgaard, K. Mølmer, J. J. L. Morton and P. Bertet, Reaching the quantum limit of sensitivity in electron spin resonance, Nat. Nanotechnol., 2016, 110(3), 253-257, DOI: 10.1038/nnano.2015.282, ISSN 1748-3395.

$15 \mathrm{~J}$. McCord, Progress in magnetic domain observation by advanced magneto-optical microscopy, J. Phys. D: Appl. Phys., 2015, 480(33), 333001, DOI: 10.1088/0022-3727/48/ $33 / 333001$.

16 H. Zheng, Z. Sun, G. Chatzidrosos, C. Zhang, K. Nakamura, H. Sumiya, T. Ohshima, J. Isoya, J. Wrachtrup, A. Wickenbrock and D. Budker, Microwave-free vector magnetometry with nitrogen-vacancy centers along a single axis in diamond, Phys. Rev. Appl., 2020, 13, 044023, DOI: 10.1103/PhysRevApplied. 13.044023.

17 T. P. Rakitzis, P. C. Samartzis, R. L. Toomes, T. N. Kitsopoulos, A. Brown, G. G. Balint-Kurti, O. S. Vasyutinskii and J. A. Beswick, Spin-polarized hydrogen atoms from molecular photodissociation, Science, 2003, 3000(5627), 1936-1938, DOI: $10.1126 /$ science.1084809, ISSN 00368075.

18 D. Sofikitis, L. Rubio-Lago, L. Bougas, A. J. Alexander and T. P. Rakitzis, Laser detection of spin-polarized hydrogen from $\mathrm{HCl}$ and $\mathrm{HBr}$ photodissociation: Comparison of $\mathrm{H}$ - and halogen-atom polarizations, J. Chem. Phys., 2008, 1290(14), 144302, DOI: 10.1063/1.2989803.

19 T. P. Rakitzis, Pulsed-laser production and detection of spin-polarized hydrogen atoms, ChemPhysChem, 2004, 5, 1489-1494, DOI: 10.1002/cphc.200400108.

20 D. Sofikitis, C. S. Kannis, G. K. Boulogiannis and T. P. Rakitzis, Ultrahigh-density spin-polarized $\mathrm{H}$ and D observed via magnetization quantum beats, Phys. Rev. Lett., 2018, 121, 083001, DOI: 10.1103/PhysRevLett.121.083001.

21 A. K. Spiliotis, M. Xygkis, M. E. Koutrakis, K. Tazes, G. K. Boulogiannis, C. S. Kannis, G. E. Katsoprinakis, D. Sofikitis and T. P. Rakitzis, Ultrahigh-density spin-polarized hydrogen isotopes from the photodissociation of hydrogen halides: new applications for laser-ion acceleration, magnetometry, and polarized nuclear fusion, Light: Sci. Appl., 2021, 100(1), 35, DOI: 10.1038/s41377-021-00476-y, ISSN 20477538.

22 D. Sofikitis, P. Glodic, G. Koumarianou, H. Jiang, L. Bougas, P. C. Samartzis, A. Andreev and T. P. Rakitzis, Highly nuclear-spin-polarized deuterium atoms from the UV 
photodissociation of deuterium iodide, Phys. Rev. Lett., 2017, 118, 233401, DOI: 10.1103/PhysRevLett.118.233401.

23 A. Spiliotis, Ultrahigh-Density Spin-Polarized Hydrogen Isotopes from the Photodissociation of Hydrogen Halides: Production, Detection, and Applications in Magnetometry, PhD thesis, University of Crete, 2020.
24 E. Steffens and W. Haeberli, Polarized gas targets, Rep. Progr. Phys., 2003, 660(11), 1887-1935, DOI: 10.1088/0034-4885/66/11/r02.

25 S. Blanes, F. Casas, J. A. Oteo and J. Ros, The magnus expansion and some of its applications, Phys. Rep., 2009, 4700(5), 151-238, DOI: 10.1016/j.physrep.2008.11.001, ISSN 0370-1573. 